PROCEEDINGS OF THE

AMERICAN MATHEMATICAL SOCIETY

Volume 127, Number 5, Pages 1379-1385

S 0002-9939(99)04606-7

Article electronically published on January 28, 1999

\title{
A DAUNS-HOFMANN THEOREM FOR TAF-ALGEBRAS
}

\author{
D. W. B. SOMERSET
}

(Communicated by Dale Alspach)

\begin{abstract}
Let $A$ be a TAF-algebra, $Z(A)$ the centre of $A, \operatorname{Id}(A)$ the ideal lattice of $A$, and $\operatorname{Mir}(A)$ the space of meet-irreducible elements of $\operatorname{Id}(A)$, equipped with the hull-kernel topology. It is shown that $\operatorname{Mir}(A)$ is a compact, locally compact, second countable, $T_{0}$-space, that $\operatorname{Id}(A)$ is an algebraic lattice isomorphic to the lattice of open subsets of $\operatorname{Mir}(A)$, and that $Z(A)$ is isomorphic to the algebra of continuous, complex functions on $\operatorname{Mir}(A)$. If $A$ is semisimple, then $Z(A)$ is isomorphic to the algebra of continuous, complex functions on $\operatorname{Prim}(A)$, the primitive ideal space of $A$. If $A$ is strongly maximal, then the sum of two closed ideals of $A$ is closed.
\end{abstract}

\section{INTRODUCTION}

In this paper we show that certain useful properties of the ideal lattice of a $\mathrm{C}^{*}$-algebra have analogues for the ideal lattice of a TAF-algebra. Indeed if the properties are formulated in the correct way, the analogues are exact.

For any Banach algebra $A$, let $\operatorname{Prim}(A)$ be the space of primitive ideals of $A$ equipped with the hull-kernel topology, let $\operatorname{Id}(A)$ be the lattice of closed, twosided ideals of $A$, and let $Z(A)$ be the centre of $A$. If $A$ is a unital $\mathrm{C}^{*}$-algebra, then $\operatorname{Prim}(A)$ is a compact, locally compact, second countable $T_{0}$-space, $\operatorname{Id}(A)$ is a continuous, distributive lattice isomorphic to the lattice of open subsets of $\operatorname{Prim}(A)$, and $Z(A)$ is isomorphic to the algebra of continuous, complex functions on $\operatorname{Prim}(A)$ (the Dauns-Hofmann Theorem). If $A$ is separable, the primitive ideals of $A$ are precisely the prime elements of the lattice $\operatorname{Id}(A)$ (an element $p$ in a lattice $L$ is prime if whenever $x, y \in L$ with $x \wedge y \leq p$ either $x \leq p$ or $y \leq p)$, and if $A$ is an $\mathrm{AF}-\mathrm{C}^{*}$-algebra, $\operatorname{Id}(A)$ is an algebraic lattice.

Now let $A$ be a TAF-algebra (always assumed to be unital) and let $\operatorname{Mir}(A)$ be the space of closed, meet-irreducible ideals of $A$ (an ideal $I \in I d(A)$ is meetirreducible if it cannot be written as the intersection of two strictly larger closed ideals), equipped with the hull-kernel topology. In this paper we show that $\operatorname{Mir}(A)$ is a compact, locally compact, second countable $T_{0}$-space, that $\operatorname{Id}(A)$ is an algebraic lattice isomorphic to the lattice of open subsets of $\operatorname{Mir}(A)$, and that $Z(A)$ is isomorphic to the algebra of continuous, complex functions on $\operatorname{Mir}(A)$. If $A$ is semisimple, then $Z(A)$ is isomorphic to the algebra of continuous, complex functions on $\operatorname{Prim}(A)$. As an application we show that if $A$ is a strongly maximal TAF-algebra, then the sum of two closed ideals of $A$ is closed.

Received by the editors December 20, 1996 and, in revised form, May 13, 1997 and August 7, 1997.

1991 Mathematics Subject Classification. Primary 46K50, 47D25.

(C)1999 American Mathematical Society 
We have chosen to stress the meet-irreducibility of the elements of $\operatorname{Mir}(A)$, because this is the notion already in use in the ideal theory of TAF-algebras (see [7] for example, and [2] where it is shown that for strongly maximal TAF-algebras all meet-irreducible ideals arise as kernels of nest representations). $I d(A)$ is, however, a distributive lattice [3, Prop. 28], so the meet-irreducible elements of $\operatorname{Id}(A)$ coincide with the prime elements of $\operatorname{Id}(A)$ [4, I.3.12]. Thus our results correspond exactly to those for separable $\mathrm{C}^{*}$-algebras. It should be noted, however, that although every closed prime ideal of $A$ is a prime element of $\operatorname{Id}(A)$, the converse does not hold in general. For example, the ideal $\{0\}$ in the TAF-algebra $T_{2}$ of upper triangular, two-by-two complex matrices is a prime element of $\operatorname{Id}\left(T_{2}\right)$ but not a prime ideal.

Let us now give some definitions. Let $C$ be a unital AF-C*-algebra with a masa (maximal, abelian, self-adjoint subalgebra) $D$, and let $A$ be a closed subalgebra of $C$ containing $D$. Define the normalizer $N_{D}(A)$ of $D$ in $A$ by

$$
N_{D}(A)=\left\{w \in A: w \text { is a partial isometry, } w D w^{*} \subseteq D \text {, and } w^{*} D w \subseteq D\right\} .
$$

Suppose that there exists a sequence $\left\{C_{n}\right\}$ of finite-dimensional $\mathrm{C}^{*}$-algebras, such that $C=\overline{\bigcup_{n=1}^{\infty} C_{n}}$ and $D=\overline{\bigcup_{n=1}^{\infty} D_{n}}$, where $D_{n}=D \cap C_{n}$ for each $n$. If each $D_{n}$ is a masa in $C_{n}$, and if $N_{D_{n}}\left(C_{n}\right) \subseteq N_{D_{n+1}}\left(C_{n+1}\right)$ for each $n$, then $D$ is a canonical masa in $C$. Recall that a TAF-algebra $A$ is a closed subalgebra of a unital AF-C*algebra $C$ such that $D=A \cap A^{*}$ is a canonical masa in $C$. If $A$ is a TAF-algebra, then, with the notation above (which we fix, from now on), $A=\overline{\bigcup_{n=1}^{\infty} A_{n}}$, where $A_{n}=A \cap C_{n}$. For convenience we may choose a system of matrix units for each $C_{n}$ such that the matrix units in $C_{n}$ are sums of matrix units in $C_{n+1}$, and the self-adjoint matrix units are in $D_{n}$ [9]. A TAF-algebra $A$ is strongly maximal if $A+A^{*}$ is dense in $C$; see $[9,7.8]$.

Each closed ideal $I$ of a TAF-algebra $A$ is inductive, in the sense that $I=$ $\overline{\bigcup_{n=1}^{\infty}\left(I \cap A_{n}\right)}[8,2.2]$. In fact $I$ is generated by the matrix units which it contains. Furthermore, if $\left(I_{n}\right)$ is a sequence with each $I_{n} \in \operatorname{Id}\left(A_{n}\right)$, and if $I=\overline{\bigcup_{n=1}^{\infty} I_{n}}$, then $I \cap A_{n}=I_{n}$ if and only if $I_{n+1} \cap A_{n}=I_{n}$ for all $n[8,2.6]$. Let $\operatorname{Max}(A)$ be the space of maximal ideals of $A$ with the hull-kernel topology. Then each $M \in \operatorname{Max}(A)$ is the kernel of a character on $A$, and the map $M \rightarrow M \cap D$ defines a homeomorphism from $\operatorname{Max}(A)$ onto $X$, the maximal ideal space of $D[6]$. We shall identify these two spaces. The spectrum $R(A)$ of $A$ is the topological binary relation on $X$ such that $(x, y) \in R(A)$ if there is a $w \in N_{D}(A)$ such that $w d w^{*}(x)=d(y)$ for all $d \in D$. The discussion in Chapter 5 of [9] shows that if $w \in N_{D}(A)$ and $w w^{*}(x) \neq 0$, then there exists $y \in X$ such that $w d w^{*}(x)=d(y)$ for all $d \in D$.

Recall also that if $x$ and $y$ are elements in a complete lattice $L$ (such as $\operatorname{Id}(A)$ ), then $x$ is way-below $y$, written $x \ll y$, if whenever $S$ is an increasing net in $L$ with $y \leq \sup S$ there is an element $s \in S$ such that $x \leq s$; see [4]. If for each $y \in L$, $y=\sup \{x \in L: x \ll y\}$, then $L$ is called a continuous lattice. An element $x$ is said to be compact if $x \ll x$, and a complete lattice $L$ is said to be algebraic if each element of $L$ is the supremum of the compact elements which it dominates. Clearly algebraic lattices are continuous.

\section{Results}

We begin by showing that the lattice of closed ideals of a TAF-algebra is algebraic, and drawing some conclusions. 
Theorem 1. Let $A$ be a TAF-algebra. Then $\operatorname{Id}(A)$ is a countably-generated, distributive, algebraic lattice. If $I \in I d(A)$ and $B=A / I$, then $\operatorname{Id}(B)$ is a countablygenerated, distributive, continuous lattice.

Proof. Suppose that $k$ is fixed and that $a \in A_{k}$. Let $I_{a}$ denote the smallest closed ideal of $A$ containing $a$. We show that $I_{a}$ is a compact element of $\operatorname{Id}(A)$. Let $\left(I_{\beta}\right)$ be an inceasing net in $I d(A)$ with limit $I=\overline{\bigcup_{\beta} I_{\beta}} \supseteq I_{a}$. Then for each $n,\left(I_{\beta} \cap A_{n}\right)$ is an increasing net of ideals in $A_{n}$, with supremum $I_{n}$, let us say. But $\operatorname{Id}\left(A_{n}\right)$ is finite, because $A_{n}$ contains the diagonal $D_{n}$, so each ideal of $A_{n}$ is generated by the matrix units it contains. Hence eventually $I_{\beta} \cap A_{n}=I_{n}$. Furthermore it is clear that $I_{n+1} \cap A_{n}=I_{n}$, from which it follows that $I=\overline{\bigcup_{n=1}^{\infty} I_{n}}$, and that $I \cap A_{n}=I_{n}$ $[8,2.6]$. Thus eventually $a \in I_{\beta} \cap A_{k}=I_{k}$. Hence eventually $I_{a} \subseteq I_{\beta}$, so $I_{a}$ is a compact element of $\operatorname{Id}(A)$. Thus each closed ideal of $A$ is the supremum of the compact ideals which it contains, which means that $\operatorname{Id}(A)$ is an algebraic lattice. Now let $K$ be the family of closed ideals of $A$ generated by the ideals of the various subalgebras $A_{n}$. Then $K$ is countable, since $\operatorname{Id}\left(A_{n}\right)$ is finite for each $n$, and $K$ is a generating set for $\operatorname{Id}(A)$, since each ideal of $A$ is inductive. The distributivity of $\operatorname{Id}(A)$ was proved in [3, Prop. 28].

The second statement follows immediately from the fact that a quotient of an algebraic lattice is a continuous lattice (not necessarily algebraic) [4, I.2.7(iii)].

For a Banach algebra $A$, let $\operatorname{Mir}(A)$ denote the set of meet-irreducible elements of the lattice $\operatorname{Id}(A)$. If $\operatorname{Id}(A)$ is distributive, these are the same as the prime elements of the lattice $\operatorname{Id}(A)$ [4, I.3.12], so $\operatorname{Mir}(A)$ can be equipped with the hullkernel topology, which is a $T_{0}$-topology. The next result follows from Theorem 1 by standard lattice theory [4, V.5.5].

Theorem 2. Let $A$ be a TAF-algebra, let $I$ be a closed ideal of $A$, and let $B=A / I$. Then $\operatorname{Mir}(B)$ is compact, locally compact, and second countable, and $\operatorname{Id}(B)$ is isomorphic to the lattice of open subsets of $\operatorname{Mir}(B)$. In particular every closed ideal of $B$ is the intersection of the closed, meet-irreducible ideals which contain it.

It follows from Theorem 2, by [4, I.3.43.8], that $\operatorname{Mir}(B)$ is a Baire space.

The second statement of Theorem 2 was proved, for strongly maximal TAFalgebras, in [2] (see also [7]).

Since meet-irreducible ideals need not be prime ideals, as we have observed, it is interesting to know which ideals are equal to the intersection of the closed prime ideals which contain them. Recall that an ideal is semiprime if it is the intersection of the (not necessarily closed) prime ideals which contain it.

Corollary 3. Let A be a TAF-algebra, and let I be a closed ideal of A. Then I is the intersection of the closed, prime ideals of $A$ which contain it if and only if $I$ is semiprime.

Proof. The necessity is trivial. Conversely, if $I$ is a semiprime ideal, then $A / I$ is a semiprime ring, so $[11,3.7(\mathrm{~b})]$, applied to the countably-generated, continuous lattice $\operatorname{Id}(A / I)$, shows that $\{0\}$ is an intersection of closed, prime ideals of $A / I$. This means that $I$ is the intersection of the closed, prime ideals of $A$ which contain it.

As Hudson has observed, Corollary 3 implies that every closed, semiprime ideal of a TAF-algebra $A$ must contain the Jacobson radical (that is, the intersection of 
the primitive ideals of $A$ ). This is because every closed, prime ideal of $A$ contains the Jacobson radical [5, Theorem 4.2]. There is evidence to suggest that every closed, semiprime ideal in a TAF-algebra is semisimple (that is, an intersection of primitive ideals). The next result shows one case in which this holds. Recall that the prime radical of a ring is the intersection of its prime ideals.

Corollary 4. Let $A$ be a TAF-algebra and let $L$ be the closure of the prime radical of $A$. Then $L$ is equal to the Jacobson radical of $A$ if and only if $L$ is semiprime.

Proof. Every primitive ideal of a Banach algebra is closed and prime, so $L$ is automatically contained in the Jacobson radical of $A$. If $L$ is equal to the Jacobson radical, then $L$ is semiprime, because the Jacobson radical is semiprime. Conversely, if $L$ is semiprime, then $L$ contains the Jacobson radical, by Hudson's observation above, so $L$ is equal to the Jacobson radical.

Another characterization of the coincidence of $L$ and the Jacobson radical is given in [5, 4.4]. Examples 4.8 and 4.9 of [5] show that $L$ need not equal the Jacobson radical, and hence need not be semiprime.

We now use Theorem 2 to show that the sum of two closed ideals in a TAFalgebra is closed, provided at least that the algebra is strongly maximal. The question of whether this is so has been open for some time. We begin with a general proposition, using an idea of Peter Dixon's [1].

Let $B$ be a Banach algebra. An element $P \in \operatorname{Id}(B)$ is said to be a primal element if whenever $\left\{I_{1}, I_{2}, \ldots, I_{n}\right\}$ is a finite subset of $\operatorname{Id}(B)$ with $I_{1} \cap I_{2} \cap \cdots \cap I_{n}=\{0\}$, then $I_{i} \subseteq P$ for at least one $i \in\{1, \ldots, n\}$. If $B$ is a quotient of a TAF-algebra, then $\operatorname{Id}(B)$ is distributive, as we have observed, so the meet-irreducible elements of $\operatorname{Id}(B)$ are prime elements of $\operatorname{Id}(B)$, and hence primal elements.

Proposition 5. Let $B$ be a Banach algebra, and suppose that $Y$ is a family of primal elements of $\operatorname{Id}(B)$ such that the seminorm

$$
\|b\|^{\prime}=\sup \{\|b+P\|: \quad P \in Y\}
$$

is a norm on $B$ equivalent to the original norm. If $I$ and $J$ are closed ideals of $B$ with $I \cap J=\{0\}$, then $I+J$ is closed.

Proof. Let $\left(x_{n}+y_{n}\right)$ be a Cauchy sequence in $I+J$, with $x_{n} \in I$ and $y_{n} \in J$ for all $n$. Let $h^{c}(I)=\{P \in Y: P \nsupseteq I\}$. Since each $P \in Y$ is a primal element, if $P \in h^{c}(I)$, then $P \supseteq J$. Hence for any $m$ and $n$,

$$
\begin{aligned}
\left\|\left(x_{n}+y_{n}\right)-\left(x_{m}+y_{m}\right)\right\| & \geq \sup \left\{\left\|\left(x_{n}+y_{n}\right)-\left(x_{m}+y_{m}\right)+P\right\|: P \in h^{c}(I)\right\} \\
& =\sup \left\{\left\|\left(x_{n}-x_{m}\right)+P\right\|: \quad P \in h^{c}(I)\right\} \\
& =\sup \left\{\left\|\left(x_{n}-x_{m}\right)+P\right\|: P \in Y\right\} \\
& =\left\|x_{n}-x_{m}\right\|^{\prime} .
\end{aligned}
$$

Thus $\left(x_{n}\right)$ is a Cauchy sequence in $I$, since $\|\cdot\|^{\prime}$ is equivalent to the original norm, so there exists $x \in I$ with $x_{n} \rightarrow x$. Likewise there exists $y \in J$ such that $y_{n} \rightarrow y$. Hence $x_{n}+y_{n} \rightarrow x+y \in I+J$ as required.

We now use the fact that if $A$ is a strongly maximal TAF-algebra and $I \in I d(A)$, then for $a \in A,\|a+I\|=\sup \{\|a+P\|: P \in \operatorname{Mir}(A), P \supseteq I\}[2,6.3]$. Hence if $B=$ $A / I$, with the quotient norm, and $b \in B$, then $\|b\|=\sup \{\|b+P\|: P \in \operatorname{Mir}(B)\}$. 
Theorem 6. Let $A$ be a strongly maximal TAF-algebra, and let $I, J \in I d(A)$. Then $I+J$ is closed.

Proof. Set $K=I \cap J$, and let $B=A / K$ with the quotient norm. Set $I^{\prime}=I / K$ and $J^{\prime}=J / K$. Then $I^{\prime} \cap J^{\prime}=\{0\}$, and $X=\operatorname{Mir}(B)$ satisfies the requirements of Proposition 5 , as observed above, so $I^{\prime}+J^{\prime}$ is closed in $B$. Hence $I+J$ is closed in $A$, being the inverse image of $I^{\prime}+J^{\prime}$ under the quotient map.

There are examples in [13] of pairs of closed ideals in the disc algebra whose sum is not closed.

We turn now to consider the centre of a TAF-algebra $A$, proving an analogue of the Dauns-Hofmann Theorem. Let $C(\operatorname{Mir}(A))$ and $C(\operatorname{Prim}(A))$ be the algebras of continuous, complex functions on $\operatorname{Mir}(A)$ and $\operatorname{Prim}(A)$ respectively, with the uniform norm. We shall show that $Z(A)$ is always isomorphic to $C(\operatorname{Mir}(A))$, and is isomorphic to $C(\operatorname{Prim}(A))$ if $A$ is semisimple.

Proposition 7. Let $A$ be a TAF-algebra. Then

$$
Z(A)=\{d \in D: d(x)=d(y) \text { for all }(x, y) \in R(A)\} .
$$

Proof. First note that $Z(A) \subseteq D$, because $D$ is a masa in $C$, and hence a maximal abelian subalgebra of $C$ by Fuglede's Theorem. Suppose that $z \in Z(A)$ and that $(x, y) \in R(A)$. Let $w \in N_{D}(A)$ be such that $w d w^{*}(x)=d(y)$ for all $d \in D$. Then $w w^{*}(x)=1$, so $z(y)=w z w^{*}(x)=z w w^{*}(x)=z(x)$. Hence $z$ has the required property.

Conversely, suppose that $z \in D$ and $z(x)=z(y)$ for all $(x, y) \in R(A)$. It is enough to show that $z$ commutes with every matrix unit $w \in A$. Set $q=w w^{*} \in D$, and let $x \in X$. If $q(x)=0$, then $w z w^{*}(x)=w z w^{*} q(x)=0=z q(x)$. If $q(x)=1$, let $y \in X$ be the character such that $w d w^{*}(x)=d(y)$ for all $d \in D$. Then $(x, y) \in R(A)$ so $w z w^{*}(x)=z(y)=z(x)=z q(x)$, by assumption on $z$. Hence $w z w^{*}=z q$, so $w z=w q z=w z q=w z w^{*} w=z q w=z w$. Thus $z \in Z(A)$.

Let $T$ be a topological space, and let $x, y \in T$. We say that $x \sim y$ in $T$ if $x$ and $y$ cannot be separated by disjoint, open subsets of $T$. If $f$ is a continuous function from $T$ into a Hausdorff space and $x \sim y$, then $f(x)=f(y)$.

Lemma 8. Let $A$ be a TAF-algebra, and let $x, y \in X \subseteq M \operatorname{ir}(A)$. If $(x, y) \in R(A)$, then $x \sim y$ in $\operatorname{Mir}(A)$.

Proof. Suppose that $x \nsim y$. Then by definition of the hull-kernel topology, there exist $I, J \in I d(A)$ such that $x \nsupseteq I, y \nsupseteq J$, and $I \cap J=\{0\}$. Let $w$ and $v$ be matrix units with $w \in I \backslash x$ and $v \in J \backslash y$. Then $w A v=0$, so $p A q=q A^{*} p=0$, where $p=w^{*} w$ and $q=v v^{*}$. Suppose, for a contradiction, that there exists $u \in N_{D}(A)$ such that $\left(u d u^{*}\right)(x)=d(y)$ for all $d \in D$. Then $\left(u q u^{*}\right)(x)=q(y) \neq 0$, so $\left(u q u^{*} p\right)(x)=\left(u q u^{*}\right)(x) p(x) \neq 0$, so $u q u^{*} p \neq 0$. But $q u^{*} p \in q A^{*} p=0$, a contradiction. Thus $(x, y) \notin R(A)$.

Lemma 9. Let $A$ be a TAF-algebra. If $I \in \operatorname{Mir}(A)$, then $I \cap Z(A)$ is a maximal ideal of $Z(A)$.

Proof. Suppose, for a contradiction, that $M=I \cap Z(A)$ is not a maximal ideal of $Z(A)$. Proposition 7 shows that $Z(A)$ is a $\mathrm{C}^{*}$-algebra, so there must exist $z, w, p, q \in$ $Z(A) \backslash M$ such that $p z=z, q w=w$, and $p q=0$. Let $J$ and $K$ be the closed ideals of $A$ generated by $z$ and $w$ respectively. Then $p j=j$ for all $j \in J$, and $q k=k$ for 
all $k \in K$. Hence if $a \in J \cap K, 0=p q a=p a=a$. Since $J, K \nsubseteq \nsubseteq I, I$ is not a prime element of $\operatorname{Id}(A)$. But meet-irreducible elements are prime in a distributive lattice, as we have already observed, so we have a contradiction.

Theorem 10. Let $A$ be a TAF-algebra. Then $Z(A) \cong C(\operatorname{Mir}(A))$.

Proof. By Lemma 9 we can, for each $z \in Z(A)$, define a complex-valued function $\hat{z}$ on $\operatorname{Mir}(A)$ by $\hat{z}(P)=z+P(P \in \operatorname{Mir}(A))$, and $\hat{z} \in C(\operatorname{Mir}(A))$ by [12, 3.3.4, 4.10]. It is clear that the map $\Theta: z \mapsto \hat{z}(z \in Z(A))$ is an injective homomorphism from $Z(A)$ into $C(\operatorname{Mir}(A))$, and it is easy to see that $\Theta$ is an isometry. We must show that $\Theta$ is a surjection. Let $f \in C(\operatorname{Mir}(A))$. Then $\left.f\right|_{X}$ is continuous on $X$, so there exists $d \in D$ such that $f(x)=d(x)$ for all $x \in X$. Suppose that $(x, y) \in R(A)$. Then $x \sim y$ in $\operatorname{Mir}(A)$ by Lemma 8, which implies that $f(x)=f(y)$, so $d(x)=d(y)$. Thus $d \in Z(A)$ by Proposition 7. Let $g=f-\hat{d} \in C(\operatorname{Mir}(A))$. Then $g(x)=0$ for all $x \in X$. But $P \subseteq Q \Rightarrow P \sim Q \Rightarrow g(P)=g(Q)$ for $P, Q \in \operatorname{Mir}(A)$, so $g=0$. We have shown that $\Theta$ is a surjection.

Now suppose that $A$ is semisimple. Then $\operatorname{Prim}(A)$ is dense in $\operatorname{Mir}(A)$ (see [10, 2.2(ii)] for example), which implies that for $x$ and $y$ in $X, x \sim y$ in $\operatorname{Prim}(A)$ if and only if $x \sim y$ in $\operatorname{Mir}(A)$.

Corollary 11. Let $A$ be a semisimple TAF-algebra. Then $Z(A) \cong C(\operatorname{Prim}(A))$.

Proof. Let $\Theta$ be the isomorphism from $Z(A)$ to $C(\operatorname{Mir}(A))$ obtained in Theorem 10 , and define $\Theta^{\prime}: Z(A) \rightarrow C(\operatorname{Prim}(A))$ by $\Theta^{\prime}(z)=\left.\hat{z}\right|_{\operatorname{Prim}(A)}(z \in Z(A), P \in$ $\operatorname{Prim}(A))$. The density of $\operatorname{Prim}(A)$ in $\operatorname{Mir}(A)$ implies that $\Theta^{\prime}$ is an isomorphism onto its range. We must show that $\Theta^{\prime}$ is surjective. Let $f \in C(\operatorname{Prim}(A))$. Then $\left.f\right|_{X}$ is continuous on $X$, so there exists $d \in D$ such that $f(x)=d(x)$ for all $x \in X$. If $(x, y) \in R(A)$, then $x \sim y$ in $\operatorname{Mir}(A)$ by Lemma 8 , so $x \sim y$ in $\operatorname{Prim}(A)$, by the remark above. The proof now proceeds as in Theorem 10 .

The conclusion of Corollary 11 is not true for all TAF-algebras. To see this, suppose that $A$ is a non-abelian TAF-algebra whose strong and Jacobson radicals coincide; see the examples before $[5,5.6]$. Then $\operatorname{Prim}(A)=X$, by $[5,5.6]$, so $C(\operatorname{Prim}(A)) \cong D$. If $D$ were equal to $Z(A)$, then $D$ would equal $A$, because $D$ is a masa, hence a maximal abelian subalgebra by Fuglede's Theorem, in $C$. Thus $C(\operatorname{Prim}(A)) \varsubsetneqq Z(A)$.

The author is grateful to Tim Hudson and to Rob Archbold for their help with this paper.

\section{REFERENCES}

[1] P. G. Dixon, Non-closed sums of closed ideals in Banach algebras, preprint.

[2] A. P. Donsig, A. Hopenwasser, T. D. Hudson, M. P. Lamoureux, B. Solel, Meet irreducible ideals in direct limit algebras, Math. Scand., to appear.

[3] A. P. Donsig, T. D. Hudson, On the lattice of ideals of triangular AF algebras, J. Funct. Anal., 138 (1996), 1-39. MR 97e:47068

[4] G. Gierz, K. H. Hofmann, K. Keimel, J. Lawson, M. Mislove, D. S. Scott, A Compendium of Continuous Lattices, Springer-Verlag, New York, 1980. MR 82h:06005

[5] T. D. Hudson, Radicals and prime ideals in limit subalgebras of AF algebras, Quart. J. Math. Oxford (2) 48 (1997), 213-233. MR 98i:46053

[6] T. D. Hudson, E. G. Katsoulis, Primitive triangular UHF algebras, J. Funct. Anal., to appear.

[7] M. P. Lamoureux, The topology of ideals in some triangular AF algebras, J. Operator Theory 37 (1997), 91-109. CMP 97:04 
[8] J. R. Peters, Y. T. Poon, B. H. Wagner, Triangular AF algebras, J. Operator Theory, 23 (1990), 81-114. MR 91h:46102

[9] S. C. Power, Limit Algebras, Pitman Research Notes in Mathematics, No. 278, Longman, London, 1992. MR 94g:46001

[10] D. W. B. Somerset, Minimal primal ideals in Banach algebras, Math. Proc. Camb. Phil. Soc., 115 (1994), 39-52. MR 94k:4609

[11] _ Minimal primal ideals in rings and Banach algebras, J. Pure Appl. Alg., to appear.

[12] _ Ideal spaces of Banach algebras, Proc. London Math. Soc., to appear.

[13] D. A. Stegenga, Ideals in the disk algebra, J. Funct. Anal. 25 (1977), 335-337. MR 58:2307

Department of Mathematical Sciences, University of Aberdeen, AB24 Ue United KINGDOM

E-mail address: ds@maths.abdn.ac.uk 